\title{
A Closer Look at the Brain in 3D Using FIB-SEM
}

C. Shan $\mathrm{Xu}^{*}$ and Harald Hess*

* Howard Hughes Medical Institute, Janelia Farm Research Campus, 19700 Helix Drive, Ashburn, VA 20147

A complete reconstruction of a brain wiring diagram puts high demand on image resolution [1]. Although electron micrograph provides sufficient lateral resolution compared to fluorescence images, traditional transmission electron microscopy (TEM) of sections has fundamental limitations along the z-axis. On the other hand, Focus ion beam (FIB) technology, initially developed for semiconductor industry, can be used as a molecular machining tool. Combined with block face imaging, FIB-SEM produces 3D images with isotropic voxel at adequate resolution $(5-10 \mathrm{~nm})$ for varieties of biological samples [2].

However, there are many challenges for FIB-SEM to acquire 3D images with a large volume. SEM is intrinsically slower compared to TEM. For example, a 100 micron cube consists of 8 Tera-voxels at $5 \mathrm{~nm}$ resolution along three dimensions. The 24/7 non-stop milling and imaging cycle takes weeks to months to complete. Common FIB sources are only capable to operate for a few days continuously before a reheat is needed therefore creates interruptions. We managed to overcome the throughput limitations of SEM and developed smooth restart procedure after an ion source reheat, so that continuous 3D images of a large volume can be obtained.

We have applied FIB-SEM technology to image the optic lobe of drosophila brain. Newly obtained 3D images have an isotropic voxel size of 5 and $7 \mathrm{~nm}$, respectively. 3D images of lamina and medulla enable visualization of previously unseen features, such as multiple complex post-synaptic density structures, in fine details. In addition to brain tissues, bacteria and cultured mammalian cells were also studied [3].

\section{References}

[1] M. Helmstaedter et al., Curr. Opin. Neurobiol. Microanal. 18 (2008) 633.

[2] G. Knott et al, J. Neurosci. 28 (2008) 2959.

[3] We would like to thank Dr. Richard Fetter, Prof. Ian Meinertzhagen, and Zhiyuan Lu for providing the brain tissue samples. 